\title{
FORNECIMENTO DE MISTURADOR DE GASES AUTOMÁTICO COM BY-PASS MANUAL PARA GERAÇÃO DE ATMOSFERAS EM FORNOS DE TRATAMENTO TÉRMICO*
}

\section{Resumo}

Tiago Ribeiro Costa ${ }^{1}$

A Praxair é uma das três maiores empresas de gases industriais do mundo, com atuação em mais de 40 países e fortemente atuante na indústria siderúrgica. A Praxair possui experiência para fornecer produtos e serviços para todos os tipos de siderúrgica. Diversas tecnologias desenvolvidas pela Empresa, como o CoJet ${ }^{\circledR}$ (sistema para injeção de jato coerente de oxigênio em fornos elétricos e conversores), Slag Splashing (espalhamento de escória nas paredes de conversores, aumentando a vida útil dos refratários), $A O D$ (processo para produção de aços especiais) e outros, encontram-se em operação comercial atualmente em diferentes partes do mundo. No Brasil, a PRAXAIR é a controladora da White Martins, maior empresa de gases industriais da América do Sul, presente em nove países do continente. Seu portfólio inclui gases atmosféricos, produção de gás carbônico, acetileno, hidrogênio, gases especiais e medicinais, misturas para soldagem, cilindros de aço sem costura, equipamentos para aplicação, transporte e armazenamento de gases. A empresa também opera uma unidade para liquefação de gás natural. Além de tecnologias e processos, a White Martins também pode fornecer soluções e equipamentos para medição, controle e injeção de gases, atendendo às rígidas normas de segurança e padrões de qualidade. O Objetivo deste trabalho é apresentar e discutir sobre a solução para o fornecimento de mistura para atmosfera de fornos de tratamento térmico através de um painel misturador de gases hidrogênio e nitrogênio. Será discutida a operação do equipamento e abordadas às características construtivas.

Palavras-chave: Hidrogênio; Tratamento térmico; Meio ambiente; recozimento; utilidades; Misturador de gases; Craqueamento de amônia; Segurança; Confiabilidade.

\section{AUTMOMATIC GAS MIXER WITH A MANUAL BY-PASS FOR GENERATION OF HEAT TREATMENT FURNACES ATMOSPHERE}

\section{Abstract}

Praxair is one of the largest industrial gases producers of the World, being present in more than 40 countries and strongly connected to the steel industry. Praxair has experience to supply products and services to all kinds of steel mills. Several technologies developed by the Company, such as CoJet ${ }^{\circledR}$ (a system to coherent oxygen jet injection in EAF and BOF furnaces), Slag Splashing (splashing slag to cover BOF furnaces walls increasing refractory wear campaign life), AOD (a process for specialty steel production) and others, are currently in commercial operation in different parts of the world. In Brazil, PRAXAIR is the owner of White Martins, the largest industrial gases company in South America, present in nine countries of the continent. Company's portfolio includes atmospheric gases, carbonic gas production, acetylene, hydrogen, specialty and health \& care gases, mixtures for welding, gas seamless steel cylinders, applications equipment, and storage and gases transportation. The Company also operates a natural gas liquefaction unit. In addition to technologies and process, Praxair / White Martins can also supply solutions for measurement, control and gases injection, according to strict safety and quality standards. The main objective of the work is to present and discuss the solution developed to supply a gaseous mixture for the heat treatment furnaces through a hydrogen and nitrogen mixer. Equipment operation and its characteristics will be discussed.

Keywords: Hydrogen; Heat Treatment; Environment; Annealing; Utilities; Gas Mixer; Ammonia Cracking; Safety, Reliability

1 Técnico e Engenheiro Mecânico, Gerente de Aplicações e Processos de Metalurgia e Siderurgia, On Site, White Martins Gases Industriais Ltda., Rio de Janeiro, RJ, Brasil.

* Contribuição técnica ao $35^{\circ}$ Seminário de Balanços Energéticos Globais e Utilidades e $29^{\circ}$ Encontro de Produtores e Consumidores de Gases Industriais, 13 a 15 de agosto de 2014, São Paulo, SP, Brasil. 


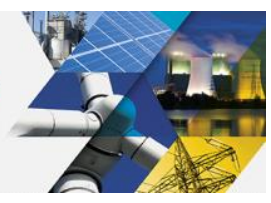

\section{INTRODUÇÃO}

O conceito do misturador de gases já vinha sendo utilizado pela White Martins em diversas aplicações e alguns misturadores menores sendo equipamentos de linha. $O$ desenvolvimento de misturadores de gases de maior porte e de projeto customizado visa atender a processos variados, volumes elevados e com demandas mais específicas de fornecimento. $O$ caso descrito no presente trabalho ilustra detalhadamente a necessidade de um projeto específico.

$\mathrm{Na}$ instalação original, havia um a planta de craqueamento de amônia para produção de atmosfera de fornos de tratamento térmico. Com a finalidade de adotar um processo com menor impacto ambiental e custos de operação e manutenção reduzidos, foi estipulado um prazo de até 4 meses para desativação da planta. Dessa forma, foi projetado um equipamento para mistura de gases contínua e automática de hidrogênio e nitrogênio. A concentração de hidrogênio é ajustável para alcançar a atmosfera requerida nos fornos, e assim desativar a planta de amônia.

Para atender ao prazo, o equipamento foi separado em 2 módulos: um módulo manual com válvulas manuais e rotâmetros para operação assistida que ficaria pronto antes do prazo limite e possibilitaria a desativação da planta de amônia, além de outro automático entregue posteriormente, com válvulas de controle automáticas, medidores de pressão e vazão que efetuam a mistura programada e monitoram as correntes indicadas em uma IHM (Interface Homem Máquina) de forma a garantir a segurança da operação. O módulo automático fica acoplado ao manual, fora projetado para ser regulado/testado ventando a mistura, e após comissionado, poderia ser alinhado ao processo sem ter que parar a operação dos fornos.

O projeto foi entregue dentro do prazo, o cliente conseguiu desativar a planta de amônia a tempo sem parar a produção e com alto grau de satisfação.

A obtenção da mistura através do Pmix (Misturador de Gases) tem como vantagens em relação ao processo anterior maior segurança, pois conta com diversos intertravamentos aliados aos analisadores de gases, dimensões reduzidas, menor demanda de manutenção, operação simplificada e não necessita de operação assistida no modo automático.

\section{MATERIAIS E MÉTODOS}

\subsection{Painel de Mistura Manual}

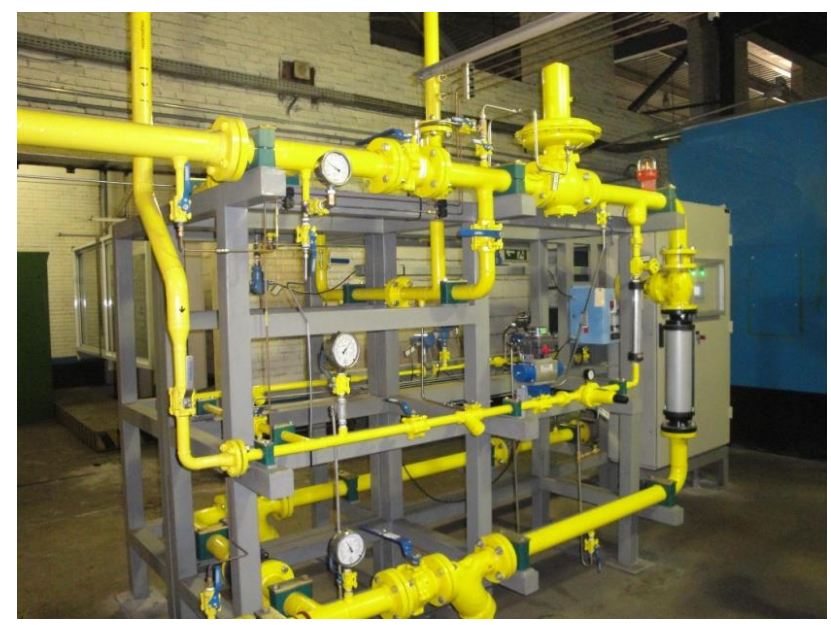

Fig. 1 Face do Painel de Mistura manual

* Contribuição técnica ao $35^{\circ}$ Seminário de Balanços Energéticos Globais e Utilidades e $29^{\circ}$ Encontro de Produtores e Consumidores de Gases Industriais, 13 a 15 de agosto de 2014, São Paulo, SP, Brasil. 


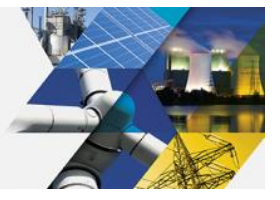

O painel de mistura manual possui instrumentos para regulagem de pressão, medição e controle de vazão de Hidrogênio e Nitrogênio de forma manual utilizando regulador de pressão auto-operado, medidores de vazão tipo rotâmetros e válvulas tipo globo, além de válvulas de bloqueio manuais tipo esfera. As pressões são monitoradas através de manômetros na entrada do equipamento para pressão de suprimento e na saída dos reguladores de pressão, para a pressão de mistura. Existe também uma válvula de bloqueio automática, escrava da análise de mistura dos gases feita por um analizador. Caso a concentração de hidrogênio se torne elevada, a válvula fecha o fornecimento de hidrogênio, deixando apenas o nitrogênio alinhado, garantindo a inertização da linha.

\subsection{Painel de Mistura Automática}

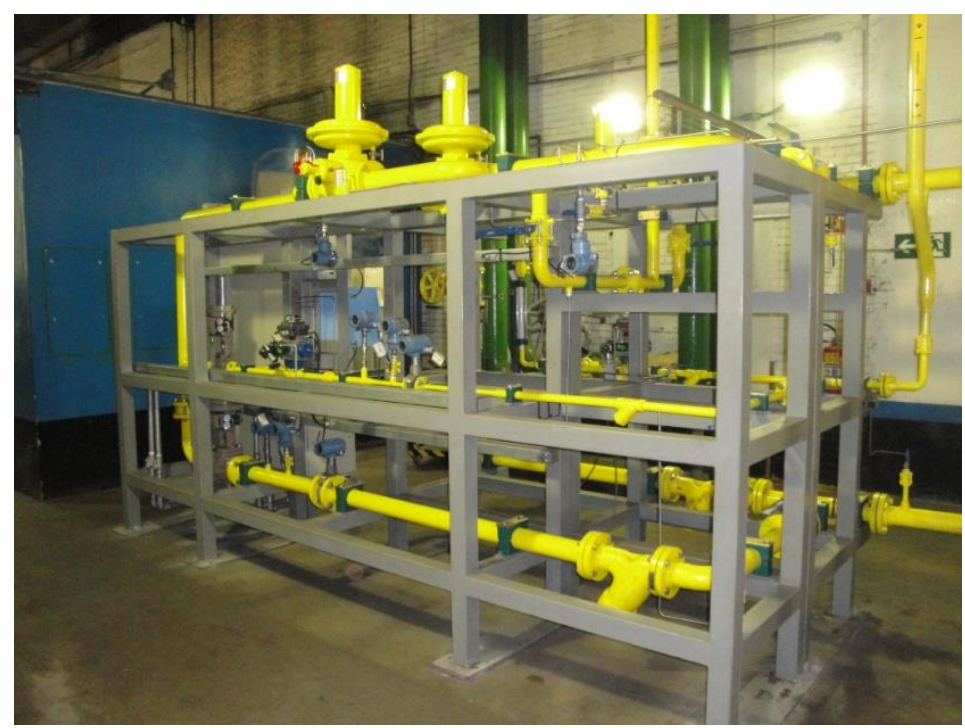

Fig. 2 Face do Painel de Mistura Automático

O painel de mistura de gases Hidrogênio e Nitrogênio é projetado para fornecer mistura contínua dentro das vazões e pressões estabelecidas.

O painel misturador automático possui instrumentos para controle de pressão e medição de vazão de Nitrogênio, medição e controle de vazão de Hidrogênio, estas isoladas através de sistema de proteção "block and bleed" (duplo bloqueio e vente), um bloco para homogeneização da mistura, um regulador de pressão manual para suprimento de mistura, uma linha derivada do suprimento de Nitrogênio com regulador de pressão para suprir as válvulas piloto operadas e um analisador de gases de concentração de Hidrogênio em Nitrogênio.

Todo o controle é feito por PLC dedicado, com ajustes dos parâmetros através de IHM local (Interface Homem-Máquina).

* Contribuição técnica ao $35^{\circ}$ Seminário de Balanços Energéticos Globais e Utilidades e $29^{\circ}$ Encontro de Produtores e Consumidores de Gases Industriais, 13 a 15 de agosto de 2014, São Paulo, SP, Brasil. 


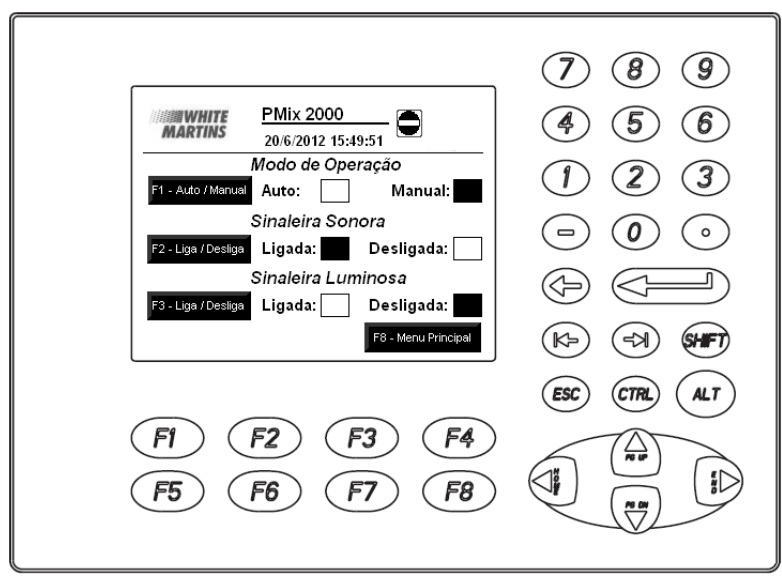

Fig. 3 Interface Homem-Máquina

\subsection{Conceito da Operação do Equipamento no Modo Automático}

A vazão de nitrogênio se dá em função do consumo de processo, ou seja, ao abrir as válvulas de bloqueio inicia-se a operação do equipamento. Para a produção da mistura, o equipamento controla a pressão de nitrogênio para estabilizar o processo e dosa a vazão de hidrogênio necessária para chegar a concentração especificada através de um cálculo. A leitura através do analisador de gases visa confirmar a equalização da mistura e também está ligado aos intertravamentos de segurança.

O painel de mistura opera de forma automática dosando o gás de menor concentração na mistura de acordo com o valor ajustado pelo operador, com setpoint de 6,5\% de Hidrogênio em Nitrogênio neste caso. Este ajuste deve ser feito através da IHM.

No painel de mistura Pmix, o valor da vazão instantânea de nitrogênio medido é enviado para o PLC através do transmissor indicador de vazão onde é corrigido com base nos valores efetivos de pressão e temperatura lidos nos transmissores indicadores de pressão e temperatura.

A vazão necessária de Hidrogênio, para garantir o percentual desejado, é calculada no PLC, com base na vazão instantânea corrigida de Nitrogênio, como indicado abaixo:

$\% \mathrm{H}_{2}=\mathrm{QH}_{2} /$ (QNitrogênio $+\mathrm{QH}_{2}$ ) onde:

$$
\mathrm{QH}_{2}=\frac{\mathrm{QN}_{2} \times \% \mathrm{H}_{2}}{\left(1-\% \mathrm{H}_{2}\right)}
$$

$$
\mathrm{QH}_{2}=\frac{Q \mathrm{~N}_{2} \times 0,10}{(1-0,10)}
$$

O controle da vazão de Hidrogênio medido é feito pela válvula de controle proporcional. Este é enviado para o PLC através do transmissor indicador de vazão. A vazão de operação do painel de mistura é determinada pelo consumo, estando limitada a vazão máxima de projeto, que representa $8 \%$ acima do valor da vazão nominal de projeto, e representa o valor máximo mensurável pelo medidor de vazão de Nitrogênio.

A válvula de bloqueio ON / OFF em conjunto com a válvula de controle são responsáveis pelo sistema "block and bleed" do Hidrogênio, evitando o retorno de Nitrogênio para a linha de Hidrogênio em caso de falhas somadas de vedação.

As pressões de suprimento de Hidrogênio e Nitrogênio são monitoradas pelos transmissores indicadores de pressão.

* Contribuição técnica ao $35^{\circ}$ Seminário de Balanços Energéticos Globais e Utilidades e $29^{\circ}$ Encontro de Produtores e Consumidores de Gases Industriais, 13 a 15 de agosto de 2014, São Paulo, SP, Brasil. 


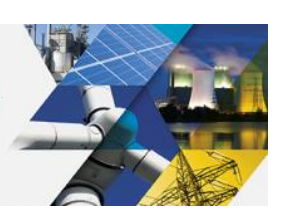

A correção de vazão dos medidores vortex com a leitura da pressão é temperatura será feita no PLC, seguindo a fórmula abaixo:

Qcorr. = Qlida $\times(($ Plida + 1.033227) $/($ Pproc + 1.033227) $) \times(($ Tproc + 273,15) $/($ Tlida +

Onde teremos: 273,15))

Qcorr. = Vazão corrigida em $\mathrm{Nm}^{3} / \mathrm{h}$

Qlida = Vazão lida em Nm³/h no transmissor correspondente (FIT 701)

Plida = Pressão lida no transmissor correspondente (PT 701)

Pproc $=$ Pressão que o vortex foi calibrado $=6,0 \mathrm{kgf} / \mathrm{cm}^{2} \mathrm{rel}$

Tproc $=$ Temperatura que o vortex foi calibrado $=21^{\circ} \mathrm{C}$

Tlida = Temperatura lida no transmissor correspondente (TT 701)

$1,0 \mathrm{~atm} .=1.033227 \mathrm{kgf} / \mathrm{cm}^{2}$

$0^{\circ} \mathrm{C}=273,15^{\circ} \mathrm{K}$ (para passar a temperatura para Kelvin)

\subsection{Conceito da Operação do Equipamento no Modo Manual}

Visando garantir o fornecimento de mistura em casos de queda de energia, manutenção do painel automático ou, no caso aqui retratado, atender ao prazo de fornecimento, dispomos de um painel de mistura de operação manual e assistida (necessário a presença de um operador para monitorar variações de processo).

Para a operação do painel de mistura manual, devemos apenas, utilizando o regulador de hidrogênio, regular a pressão para uma pressão ligeiramente maior que a de nitrogênio. Feito isso, abrimos a válvula globo de hidrogênio lentamente até termos a leitura de vazão no rotâmetro. Após é necessário abrir até alcançarmos a vazão de hidrogênio requerida.

Para saber a vazão de hidrogênio requerida se faz necessário efetuar a leitura do rotâmetro de nitrogênio e efetuar o mesmo cálculo abaixo:

$\% \mathrm{H}_{2}=\mathrm{QH}_{2} /$ (QNitrogênio $+\mathrm{QH}_{2}$ ) onde:

$$
\mathrm{QH}_{2}=\frac{\mathrm{QN}_{2} \times \% \mathrm{H}_{2}}{\left(1-\% \mathrm{H}_{2}\right)}
$$$$
Q H_{2}=\frac{Q N_{2} \times 0,10}{(1-0,10)}
$$

A operação deve ser assistida, pois ocorrendo grandes variações de consumo de mistura, se faz necessário o reajuste da dosagem de hidrogênio, observando o cálculo acima.

\subsection{Aspectos de Segurança do Equipamento}

O sistema de segurança do painel de mistura foi desenvolvido de forma que, caso ocorra falta de energia elétrica, falha nas válvulas de controle, nos medidores de vazão, analisadores ou na instrumentação, o suprimento de Hidrogênio será interrompido, ficando alinhado apenas o Nitrogênio para inertização do sistema. Em caso de falha de Nitrogênio, o sistema imediatamente bloqueia o suprimento de Hidrogênio da mesma forma.

Dentro dos aspectos de segurança dos painéis automático e manual podemos destacar:

- Análise permanente da mistura através de um analisador de gases com corte do suprimento para consumo sempre que este estiver fora da faixa especificada na operação manual.

* Contribuição técnica ao $35^{\circ}$ Seminário de Balanços Energéticos Globais e Utilidades e $29^{\circ}$ Encontro de Produtores e Consumidores de Gases Industriais, 13 a 15 de agosto de 2014, São Paulo, SP, Brasil. 


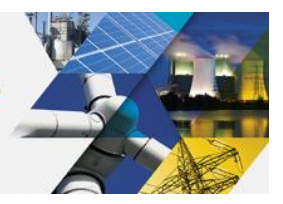

- Bloqueio automático para a linha de Hidrogênio "block and bleed" (duplo bloqueio e vente).

- Monitoração contínua do percentual de Hidrogênio na mistura, pelo PLC com corte de suprimento de Hidrogênio caso o valor esteja acima do programado.

- Sinalização local para indicação de falha com luz vermelha de alerta para além de alarme sonoro.

- Válvulas de retenção nas linhas de Hidrogênio e Nitrogênio, para evitar o retorno de um dos gases para a linha do outro, caso a pressão de um dos gases seja menor que a do outro, presentes no painel manual e automático.

- Bloqueio automático de Hidrogênio em caso de queda de energia ou baixa pressão da linha de Nitrogênio de suprimento que também é responsável por pilotar as válvulas de bloqueio automático e válvulas de controle. Todas são projetadas para em caso de falha bloquear a operação de forma segura.

- Bloqueio manual de suprimento de gases tanto para a operação manual como para a operação automática em caso de falha geral.

- Reguladoras de pressão na saída do misturador garantem a pressão de mistura dentro da especificação.

- Purga contínua de com nitrogênio dos painéis energizados.

- Toda a instrumentação com invólucro a prova de explosão ExD IIC.

- Linha de vent direcionada para fora do galpão.

\section{RESULTADOS E DISCUSSÃO}

O recozimento do cliente possuiu 40 fornos empilhadores em operação para tratamento térmico de bobinas de aço. Cada um consome cerca de $100 \mathrm{Nm}^{3} / \mathrm{h}$ de mistura de 6,5\% de Hidrogênio com balanço em Nitrogênio quando em operação a uma pressão de $1300 \mathrm{mmH} 2 \mathrm{O}$. Anteriormente, para a obtenção desta mistura o cliente detinha uma planta de craqueamento de amônia em operação. A amônia, proveniente do pólo industrial, era craqueada em reatores e tratada para utilização nos fornos. Sua operação demandava mão de obra qualificada e monitoramento permanente, principalmente devido aos riscos ambientais envolvidos, além de exigir uma manutenção elevada e minuciosa. Os pontos para possíveis vazamentos são maiores e, devido ao risco elevado de morte por intoxicação através de inalação, o monitoramento das áreas de circulação de pessoas era levado muito a sério.

O projeto já estava sendo avaliado pelo cliente, porém com a pressão do órgão ambiental local, tivemos que dar velocidade às ações.

Imediatamente após a instalação e alinhamento do misturador manual, a linha de mistura proveniente da planta de amônia foi bloqueada e raqueteada, com posterior desativação da planta.

Principalmente por ter um conceito de operação simples, o misturador não demanda grandes treinamentos de operação e logo foi absorvido pelos operadores. Sua concepção exige muito pouca manutenção, e tem menos itens sujeitos a falhas que poderiam prejudicar a operação, além da quantidade infinitamente menor de pontos para possíveis vazamentos, tais como juntas flangeadas, o que também expõe menos o operador a riscos.

A mistura é entregue de forma estável e a operação automática não demora a se adaptar a variações de consumo, mantendo sempre a mistura dentro da especificação. Com a operação no modo automático, a intervenção do operador ser faz mínima, sendo apenas para monitorar dados de operação e falhas.

* Contribuição técnica ao $35^{\circ}$ Seminário de Balanços Energéticos Globais e Utilidades e $29^{\circ}$ Encontro de Produtores e Consumidores de Gases Industriais, 13 a 15 de agosto de 2014, São Paulo, SP, Brasil. 


\section{CONCLUSÃO}

A Praxair/White Martins detém uma vasta experiência no fornecimento de gases industriais e na construção de equipamentos para operação dos mesmos em diversas aplicações, o qual usou para fabricar o equipamento nos melhores padrões de segurança e confiabilidade.

Sobretudo, trabalhamos para atender a uma necessidade em prazo de entrega e em uma solução customizada a fim de garantir a operação de nosso cliente, ao qual colocamos toda nossa experiência a disposição para desenvolver um equipamento completo. O Objetivo da Praxair/White Martins neste caso, sobretudo está no reconhecimento como uma empresa de soluções, não só se atendo ao fornecimento de gases. Este painel misturador está atualmente operando em regime de $24 \mathrm{~h}$ de operação no cliente, e já teve sua confiabilidade provada, além de ter reduzido o custo de manutenção da operação.

\section{Agradecimentos}

Agradeço aqui a toda a equipe da White Martins/Praxair ao qual trabalharam incessantemente para o bom andamento deste projeto, em especial a Lierte José Teixeira, Marden Mesquita, Rosana Sousa, Egon Lamers, Túlio Mendonça, Miguel Miyake, Nicolau Hvanov, Jorge Medeiros, Carlos Bezerra, Carlos Fogaça e Pedro Zagury.

* Contribuição técnica ao $35^{\circ}$ Seminário de Balanços Energéticos Globais e Utilidades e $29^{\circ}$ Encontro de Produtores e Consumidores de Gases Industriais, 13 a 15 de agosto de 2014, São Paulo, SP, Brasil. 